\title{
Effects of an Asian-specific nonsynonymous EDAR variant on multiple dental traits
}

\author{
Jeong-Heuy Park ${ }^{1}$, Tetsutaro Yamaguchi ${ }^{2}$, Chiaki Watanabe ${ }^{3}$, Akira Kawaguchi ${ }^{4}$, Kuniaki Haneji ${ }^{4}$, \\ Mayako Takeda ${ }^{5}$, Yong-Il Kim ${ }^{1}$, Yoko Tomoyasu ${ }^{2}$, Miyuki Watanabe ${ }^{2}$, Hiroki Oota ${ }^{6}$, Tsunehiko Hanihara ${ }^{6}$, \\ Hajime Ishida ${ }^{4}$, Koutaro Maki ${ }^{2}$, Soo-Byung Park ${ }^{1}$ and Ryosuke Kimura ${ }^{3}$
}

Dental morphology is highly diverse among individuals and between human populations. Although it is thought that genetic factors mainly determine common dental variations, only a few such genetic factors have been identified. One study demonstrated that a nonsynonymous single-nucleotide polymorphism (370V/A, rs3827760) in the ectodysplasin $A$ receptor gene (EDAR) is associated with shoveling and double-shoveling grades of upper first incisors and tooth crown size. Here, we examined the association of EDAR 370V/A with several dental characters in Korean and Japanese subjects. A meta-analysis that combined analyses of Korean and Japanese subjects revealed that the Asian-specific 370A allele is associated with an increase in the grades of shoveling and double shoveling, as previously found. We also showed a highly significant association between EDAR 370V/A genotype and crown size, especially mesiodistal diameters of anterior teeth. Moreover, we found that the 370A allele was associated with the presence of hypoconulids of lower second molars. These results indicated that the EDAR polymorphism is responsible, in part, for the Sinodonty and Sundadonty dichotomy in Asian populations, and clearly demonstrated that the EDAR polymorphism has pleiotropic effects on tooth morphology. As the 370A allele is known to be a most likely target of positive selection in Asian populations, some phenotypes associated with the variant may be 'hitchhiking phenotypes', while others may be actual targets of selection.

Journal of Human Genetics (2012) 57, 508-514; doi:10.1038/jhg.2012.60; published online 31 May 2012

Keywords: common variation; dental morphology; EDAR; polymorphisms

\section{INTRODUCTION}

Dental anthropologists and anatomists have characterized and documented many variations in human dental morphology. ${ }^{1-4}$ They have focused on differences among populations and differences among individuals within populations. Owing to their work, we now know that dental morphology is highly differentiated among human populations. Some researchers have also studied the inheritance patterns of dental traits and suggested that genetic factors are strongly involved in dental morphology., ${ }^{2,5}$

Studies of animal development have identified many genes participating in dental morphogenesis..$^{8-11}$ Additionally, studies on human genetic disorders have identified dysfunctional mutations on coding region of some genes that result in tooth malformation. ${ }^{12,13}$ However, very few examples of genetic polymorphisms associated with common dental variations have been identified. One of the examples is a variant of the ectodysplasin A receptor (EDAR) gene.
Kimura et al..$^{14}$ demonstrated that a nonsynonymous polymorphism in exon 12 of EDAR (370V/A, rs3827760) is associated with shoveling and double shoveling of incisors and crown size of teeth. This polymorphism has also been reported to have an association with morphological variation in human hair. ${ }^{15,16}$

$E D A R$ encodes a receptor for ectodysplasin A (EDA), a soluble ligand, and it is a member of the tumor necrosis factor receptor family. ${ }^{17,18}$ In humans, loss-of-function mutations of this gene result in hypohidrotic ectodermal dysplasia (MIM 129490, 224900 and 305100), which is a genetic disorder that results in congenital defects in tissues and structures of ectodermal origin such as teeth, nails, sweat glands and hair. ${ }^{19,20}$ Animal models have also suggested that the EDA/EDAR pathway regulates the development of ectodermal derivative $^{21-29}$ It is also worth noting that, in studies on human population genetics, a strong signal of positive selection in Asian populations has been observed for the EDAR $370 \mathrm{~A}$ allele. Specifically,

${ }^{1}$ Department of Orthodontics, Pusan National University Dental Hospital, Yangsan, Korea; ${ }^{2}$ Department of Orthodontics, School of Dentistry, Showa University, Tokyo, Japan; ${ }^{3}$ Transdisciplinary Research Organization for Subtropics and Island Studies, University of the Ryukyus, Okinawa, Japan; ${ }^{4}$ Department of Human Biology and Anatomy, Graduate School of Medicine, University of the Ryukyus, Okinawa, Japan; ${ }^{5}$ Department of Biological Sciences, Graduate School of Science, The University of Tokyo, Tokyo, Japan and 'Laboratory of Anatomy, Kitasato University School of Medicine, Kanagawa, Japan

Correspondence: Dr R Kimura, Transdisciplinary Research Organization for Subtropics and Island Studies, University of the Ryukyus, Uehara 207, Nishihara-cho, Nakagami-gun, Okinawa 903-0215, Japan.

E-mail: rkimura@lab.u-ryukyu.ac.jp

or Dr T Yamaguchi, Department of Orthodontics, School of Dentistry, Showa University, Kitasenzoku 2-1-1, Ota-ku, Tokyo 145-8515, Japan.

E-mail: tyamaguchi@dent.showa-u.ac.jp

Received 7 March 2012; revised 24 April 2012; accepted 4 May 2012; published online 31 May 2012 
the allele is rare in African and European populations and is associated with a long-ranged uniform haplotype in Asian populations. ${ }^{30-33}$

Many nonmetric dental variations, other than shoveling and double shoveling of incisors, have been described, and genetic factors affecting these variants have not yet been identified. Therefore, we examined several dental characters in Korean and Japanese populations to determine whether the EDAR polymorphism affects several dental characters.

\section{MATERIALS AND METHODS}

\section{Study subjects}

Plaster casts of permanent dentition were obtained from 223 Korean individuals at Pusan National University, Pusan, and from 273 Japanese individuals at Showa University Dental Hospital, Tokyo. We collected blood, buccal mucosa or saliva specimens from each of the individuals to prepare DNA samples. The Japanese subjects include patients undergoing orthodontic treatments, and these individuals might not be considered members of the general population with regard to dentition. Some Japanese participants $(n=86)$ were overlapped with those in the previous study by Kimura et al. ${ }^{14}$ and named Japanese set 1 . New Japanese participants $(n=187)$ were named Japanese set 2. We also used specimens from 73 individuals from the Sakishima Islands, the western islands of Okinawa, that had also participated in previous studies (Japanese set 3). ${ }^{14,34,35}$ All the subjects gave informed consent for their participation in this study. This study was conducted with the approval of ethical committees of the Showa University, the Pusan National University and the University of the Ryukyus.

\section{Determination of metric and nonmetric dental characteristics}

Using a sliding caliper, we measured the mesiodistal and buccolingual crown diameters of all teeth except for third molars (Figure 1a): First and second incisors (I1 and I2), canines (C), first and second premolars (P1 and P2) and first and second molars (M1 and M2) of upper (U) and lower (L) teeth. The length and width of the maxillary and mandibular dental arches were also measured: the length was defined as the distance from the line passing the lingual gingival margins of left and right Ils to the line passing the distal edges of left and right M2s, and the width was defined as the distance between the lingual gingival margins of left and right M2s. Crown size of each tooth was calculated as the geometric mean of mesiodistal and buccolingual diameters. The values over three times of the standard deviation were treated as abnormal and removed. The values of left and right homologous teeth were averaged unless only the tooth on one side was available for measurement. If the teeth on both sides were absence, the value was considered missing.

Ten nonmetric traits (shoveling of UI1, double shoveling of UI1, tuberculum dentale of UI1, UI2 and C, Carabelli's cusp of UM1 and UM2, hypocone of UM2, hypoconulid of LM2, groove pattern of LM2) were examined according to the Arizona State University dental anthropology system ${ }^{36}$ or other methods (Figures 1b-o). ${ }^{37,38}$ Grading of some nonmetric traits was modified slightly; grades were merged in some cases to avoid groups with small numbers or because some traits were ill-defined in the plaster casts. The grading methods are summarized in Supplementary Table 1 . We also scored the severity of malocclusion from grade 1 (perfect occlusion) to grade 10 (worst occlusion) according to the aesthetic component (AC) of the Index of Orthodontic Treatment Need. ${ }^{39}$

\section{SNP genotyping}

DNA was extracted from blood, buccal mucosa or saliva specimens using standard methods. The EDAR 370V/A (rs3827760) locus was genotyped using the Taqman genotyping assay (Applied Biosystems assay no: C_27497202_10; Life Technologies, Carlsbad, CA, USA). The $\chi^{2}$ test was used to identify any deviation from Hardy-Weinberg equilibrium in genotype distributions.

\section{Statistical analysis}

$T$-tests were performed to examine differences in metric data between males and females. To correct for differences between the sexes, the values of metric data were standardized into $z$ scores for each sex, and then merged them. The $z$ scores of crown sizes or diameters were averaged among all, upper, lower, anterior (I1, I2, C) or posterior (P1, P2, M1, M2) teeth to evaluate the crown size of the individual.

Any putative correlation between an EDAR genotype and a phenotype or between two phenotypes was examined using Spearman's rank correlation coefficient $(\rho)$ and Pearson's correlation coefficient $(r)$. The coefficient of determination was simply obtained as the square of the Pearson's correlation coefficient. We also observed the partial correlation coefficient between traits controlling the genotype. For a dichotomous trait, the association was also evaluated using relative risks and $\chi^{2}$ tests. The statistical tests were separately performed for each set of subjects. Then, the outcomes from different sets of subjects were combined by meta-analyses. The correlation coefficients were converted using Fisher's $r$-to- $z$ transformation, and the weighted average by the inverse variance was calculated. The relative risks were merged using the Mantel-Haenszel method.

\section{RESULTS}

The results of metric and nonmetric measurements are summarized in Supplementary Tables $2-5$. The difficulty in phenotyping greatly reduced the number of phenotyped samples in some traits. Significant sexual dimorphisms were observed, especially in the crown diameters of $\mathrm{C}$ and the maxillary and mandiblar widths. After standardizing the metric data within each sex into $z$ scores, we evaluated the crown size by averaging the $z$ scores over all teeth from each individual. Then, we examined pairwise correlations among metric and nonmetric dental traits. After meta-analyses integrating the outcomes from Korean and Japanese (Tokyo) subjects, crown size was correlated with shoveling and double shoveling of UI1, tuberculum of C, hypocone of UM2, Carabelli's cusp of UM1 and UM2, hypoconulid of LM2, and the length of maxillary and mandible, at the $P<0.05$ level (Supplementary Tables 6-8).

We successfully genotyped 222 Korean and 265 Japanese subjects for the EDAR 370V/A polymorphism, and used these genotypes for the following association study (Table 1). The genotype distribution did not deviate from the expectation of Hardy-Weinberg equilibrium in either population. The frequency of the $370 \mathrm{~A}$ allele was $86.9 \%$ and $77.5 \%$ in Korean (Pusan) and Japanese (Tokyo) subjects, respectively.

The associations of the EDAR genotype with 5 metric traits (maxillary and mandiblar lengths and widths, and crown size of all teeth) and 10 nonmetric traits were first examined using Pearson's and Spearman's correlation coefficients (Table 2). As previously reported, ${ }^{14}$ we identified significant effects of EDAR genotype on shoveling, double shoveling, and crown size in the newly examined Japanese subjects (Japanese set 2). The analysis of the Korean subjects also replicated the previous findings on shoveling and double shoveling, but not on crown size. We found that correlation coefficients between genotype and crown size were much different between the Korean and Japanese subjects. Finally, the meta-analyses showed highly significant correlations of EDAR genotype with shoveling $\left(\rho=0.28, P=5.4 \times 10^{-10}\right)$, double shoveling $(\rho=0.20$, $\left.P=9.7 \times 10^{-6}\right)$ and crown size $\left(r=0.16, P=3.7 \times 10^{-4}\right)$, which were significant after Bonferroni's correction for testing multiple traits $(P<0.05 / 15)$. When crown size were evaluated by dividing into parts, an increased correlation coefficient was observed in the mesiodistal diameters of upper anterior teeth $\left(r=0.24, P=1.3 \times 10^{-7}\right)$ (Table 2).

As the Japanese subjects were patients undergoing orthodontic treatments, we examined relation among malocclusion, crown size and EDAR genotype. As results, an index of malocclusion, AC, was significantly correlated with the average of standardized crown sizes $\left(\rho=0.12, P=9.5 \times 10^{-3}\right)$, but not with EDAR genotype $(\rho=0.03$, $P=0.59)$. Individuals with $\mathrm{AC} \geqslant 4$ showed a trend of positive 




Figure 1 Evaluations of dental characteristics. Plaster casts of maxillary and mandiblar dental arches (a). Mesiodistal (MD) and buccolingual (BL) crown diameters, and the length and width of dental arches were measured. Trace (b) and full (c, arrows) of UI1 shoveling. Absence (d) and full (e, arrows) of UII double shoveling. Absence (f) and pronounced-ridging ( $\mathbf{g}$, arrow) of $\mathbf{C}$ tuberculum dentale. Absence (h) and small cusp (i, arrow) of UM1 Carabelli's cusp. Absence (j) and large cusp (k, arrow) of UM2 hypocone. $Y(\mathbf{I}),+(\mathbf{m}), X(\mathbf{n})$ patterns of LM2 groove. Cusp numbers are shown, and cusps 2 and 4 are in contact in $Y$ pattern (I), whereas cusps 1 and 3 are in contact in $X$ pattern (n). Presence of L M2 hypoconulid (o, arrow). Right teeth are shown in ( $\mathbf{f}-\mathbf{0})$.

\section{Table 1 Genotype frequencies for EDAR 370V/A}

\begin{tabular}{|c|c|c|c|c|c|c|c|c|c|}
\hline & \multirow[b]{2}{*}{ Total } & \multirow[b]{2}{*}{$\begin{array}{l}\text { No } \\
\text { call }\end{array}$} & \multicolumn{3}{|c|}{ EDAR genotype } & \multicolumn{2}{|c|}{$\begin{array}{c}\text { Allele } \\
\text { frequency }\end{array}$} & \multicolumn{2}{|c|}{$\begin{array}{l}\text { Test for } \\
H W E^{\mathrm{a}}\end{array}$} \\
\hline & & & $\begin{array}{l}0: \\
V V\end{array}$ & $\begin{array}{l}\text { 1: } \\
V A\end{array}$ & $\begin{array}{l}2: \\
A A\end{array}$ & V & $A$ & $\chi^{2}$ & $\begin{array}{c}\mathrm{P}- \\
\text { value }\end{array}$ \\
\hline Korean & 223 & 1 & 2 & 55 & 165 & 0.133 & 0.867 & 1.25 & 0.26 \\
\hline $\begin{array}{l}\text { Japanese set } \\
1+2\end{array}$ & 273 & 8 & 15 & 89 & 161 & 0.225 & 0.775 & 0.34 & 0.56 \\
\hline
\end{tabular}

aHardy-Weinberg equilibrium. correlation between EDAR genotype and crown size in both the Korean $(n=102)$ and Japanese $(n=188)$ subjects, whereas individuals with $\mathrm{AC}<4$ (Korean: $n=119$, Japanese $n=29$ ) did not (Table 2), which indicates that individuals with malocclusion receive a larger effect of EDAR genotype on crown size than those without malocclusion.

We detected a marginally significant correlation between the EDAR genotype and the absence/presence of hypoconulid of LM2 ( $\rho=0.14$, $P=5.9 \times 10^{-3}$ ) in the meta-analysis (Table 2), which was not significant after the Bonferroni's correction for testing multiple traits. To confirm this finding, we examined the Japanese set 3, Sakishima Islanders. As the grading of this trait was dichotomous, we calculated 
Table 2 Correlations between EDAR genotypes and dental traits

\begin{tabular}{|c|c|c|c|c|c|c|c|c|c|c|}
\hline & \multicolumn{2}{|c|}{ Combined } & \multicolumn{2}{|c|}{ Korean $(\mathrm{n}=222)$} & \multicolumn{2}{|c|}{ Japanese set $1+2$} & \multicolumn{2}{|c|}{ Japanese set $1(n=84)$} & \multicolumn{2}{|c|}{ Japanese set $2(\mathrm{n}=181)$} \\
\hline & $\mathrm{R}$ & $\mathrm{P}$ & $\mathrm{R}$ & $P$ & $\mathrm{R}$ & $\mathrm{P}$ & $\mathrm{R}$ & $\mathrm{P}$ & $\mathrm{R}$ & $P$ \\
\hline \multicolumn{11}{|l|}{ Metric traits } \\
\hline Shovel UI1 & 0.28 & 5.4.E -10 & 0.26 & 8.3.E -05 & 0.30 & 1.9.E -06 & 0.25 & 2.4.E -02 & 0.32 & 2.9.E -05 \\
\hline Double shovel UI1 & 0.20 & 9.7.E -06 & 0.15 & 2.4.E -02 & 0.25 & 8.4.E -05 & 0.14 & 2.1.E -01 & 0.29 & 1.1.E -04 \\
\hline Hypoconulid LM2 & 0.14 & 5.9.E -03 & 0.10 & 1.8.E -01 & 0.20 & 9.5.E -03 & 0.28 & 2.8.E -02 & 0.12 & 2.1.E -01 \\
\hline \multicolumn{11}{|l|}{ Nonmetric traits } \\
\hline Std. crown size all & 0.16 & 3.7.E -04 & 0.02 & 8.1.E-01 & 0.28 & 4.5.E -06 & 0.36 & 7.0.E -04 & 0.22 & 2.8.E -03 \\
\hline Std. crown size upper & 0.16 & 5.7.E -04 & 0.03 & 7.1.E -01 & 0.26 & 1.6.E -05 & 0.32 & 3.5.E -03 & 0.23 & 2.5.E -03 \\
\hline Std. crown size lower & 0.14 & 1.6.E -03 & 0.00 & 1.0.E + 00 & 0.26 & 2.0.E -05 & 0.37 & 5.9.E -04 & 0.19 & 1.0.E -02 \\
\hline Std. crown size anterior & 0.19 & 2.4.E - 05 & 0.09 & 1.9.E -01 & 0.27 & 6.4.E -06 & 0.40 & 1.9.E -04 & 0.20 & 9.0.E -03 \\
\hline Std. crown size posterior & 0.12 & 8.9.E -03 & -0.05 & 5.0.E -01 & 0.25 & 3.3.E -05 & 0.31 & 3.9.E-03 & 0.22 & 3.8.E -03 \\
\hline Std. MD diameters all & 0.17 & 1.2.E -04 & 0.06 & 3.7.E -01 & 0.26 & 1.3.E -05 & 0.34 & 1.6. $E-03$ & 0.22 & 3.1.E -03 \\
\hline Std. MD diameters anterior & 0.24 & 1.3.E -07 & 0.16 & 1.5.E -02 & 0.30 & 1.0.E -06 & 0.38 & 4.3.E -04 & 0.24 & 9.4.E -04 \\
\hline Std. MD diameters posterior & 0.10 & 2.1.E -02 & -0.03 & 7.0.E -01 & 0.21 & 5.4.E -04 & 0.28 & 1.0.E -02 & 0.17 & 1.9.E -02 \\
\hline Std. BL diameters all & 0.12 & 7.4.E -03 & -0.04 & 5.9.E -01 & 0.25 & 4.0.E -05 & 0.34 & 1.5.E -03 & 0.19 & 1.2.E -02 \\
\hline Std. BL diameters anterior & 0.10 & 2.6.E -02 & 0.00 & 9.6.E -01 & 0.18 & 3.0.E -03 & 0.30 & 6.4.E -03 & 0.10 & 1.7.E -01 \\
\hline Std. BL diameters posterior & 0.11 & 1.2.E -02 & -0.07 & 3.2.E -01 & 0.26 & 1.8.E -05 & 0.32 & 2.8.E -03 & 0.22 & 3.4.E -03 \\
\hline Std. crown size all $(A C \geqslant 4)$ & 0.24 & 5.3.E -05 & 0.11 & 2.6.E -01 & 0.31 & 1.7.E -05 & & & & \\
\hline Std. crown size all $(A C<4)$ & -0.06 & 4.6.E -01 & -0.07 & 4.5.E -01 & -0.03 & 8.9.E - 01 & & & & \\
\hline
\end{tabular}

Abbreviations: AC, aesthetic component; BL, buccolingual; MD, mesiodistal; Std., standard.

$R$ denotes Spearman's and Pearson's correlation coefficients ( $\rho$ and $r$ ) for nonmetric and metric traits, respectively. The number of individuals genotyped successfully ( $n$ ) is not necessarily concordant with the number of those phenotyped successfully. Only results for traits that showed statistical significance are listed.

relative risks of the absence of hypoconulid for each genotype (Table 3). The relative risk of the $\mathrm{VA}+\mathrm{VV}$ genotypes to the AA genotype in the absence of hypoconulid was significantly more than 1 in the Japanese set $1+2(P=0.025)$ and the similar tendency was observed in the Korean subjects $(P=0.13)$. In the Japanese set 3, $\mathrm{VA}+\mathrm{VV}$ also showed a significant relative risk $\left(P=2.7 \times 10^{-3}\right)$. In the meta-analysis using data from all three populations, relative risks $(P$-values $)$ of VA, VV and VA + VV to AA were $1.68\left(6.3 \times 10^{-4}\right), 2.74$ $\left(2.2 \times 10^{-4}\right)$ and $1.84\left(3.3 \times 10^{-5}\right)$, respectively. These $P$-values were significant even after Bonferroni's correction for testing multiple traits initially examined.

The coefficients of determination, which indicates the contribution of the EDAR 370V/A to the variance of a trait, were 0.092 for shoveling of UI1, 0.040 for double shoveling of UI1, 0.021 for hypoconulid of LM2 and 0.026 for crown size of all teeth. These values suggested that there are other factors responsible for the variance of these traits. Among dental traits associated with the EDAR 370V/A, significant correlations $(P<0.05)$ were observed except for between shoveling of UI1 and hypoconulid of LM2 (Table 4). The partial correlation coefficients between the traits controlling the EDAR polymorphism were only slightly lower than the correlation coefficients (Table 4). This indicated that there are other factors commonly affecting different traits and that different combinations of factors affect different traits.

\section{DISCUSSION}

Associations of EDAR 370V/A with the shoveling, double shoveling and crown size in Japanese subjects have been described previously. ${ }^{14}$ Here, we confirmed these associations subjecting increased number of Japanese and Korean individuals. In addition, we found that the Asian-specific EDAR variant (370A) was also associated with the presence of hypoconulids of LM2. This result is parallel to a finding from previous studies demonstrating that misexpression of Edar in transgenic mice alters the diameters and the number of cusps of lower molars in a dose-dependent manner. Low doses of transgenic Edar expression reduce the number of cusps, whereas high doses increase it. ${ }^{40,41}$ The $370 \mathrm{~A}$ allele reportedly has a more potent signaling activity than the $370 \mathrm{~V}$ allele in vitro. ${ }^{42,43}$ Therefore, it is supposed that the strong activity of the $370 \mathrm{~A}$ causes a high prevalence of hypoconulid in the AA genotype. However, the possibility that another polymorphism in strong LD with EDAR $370 \mathrm{~V} / \mathrm{R}$ is responsible for the phenotypic variation has not been completely denied.

We found that the relative risk for the absence of hypoconulid was much stronger in Japanese set 3 (Sakishima Islanders) than that in the other subject sets. This may be attributed to a statistical error because of the small number of subjects: the $95 \%$ confidential interval (1.70-12.4) included the value in the meta-analysis (1.84). We also observed different outcomes of our association studies between the subject sets, especially on crown size, where there seem to be reasons, other than statistical errors. A possibility is that Korean and Japanese subjects may differ in other genetic factors that affect dental traits and interact with the EDAR genotype. The fact that the Japanese set $1+2$ includes patients for orthodontic treatments seems to have a strong influence on our association study. It has been previously reported that orthodontic patients have a larger crown size than people with normal occlusion. ${ }^{44}$ We also confirmed a significant correlation of crown size with AC. Moreover, the present analysis showed a relationship between malocclusion and the effect of EDAR genotype on enlarging crown size. It is possible that effects of some genetic factors are enhanced in orthodontic patients.

In dental anthropology, dental characters in Asian populations have been dichotomized into two categories, Sinodonty and 
Table 3 EDAR genotypes and relative risks for the absence of hypoconulids

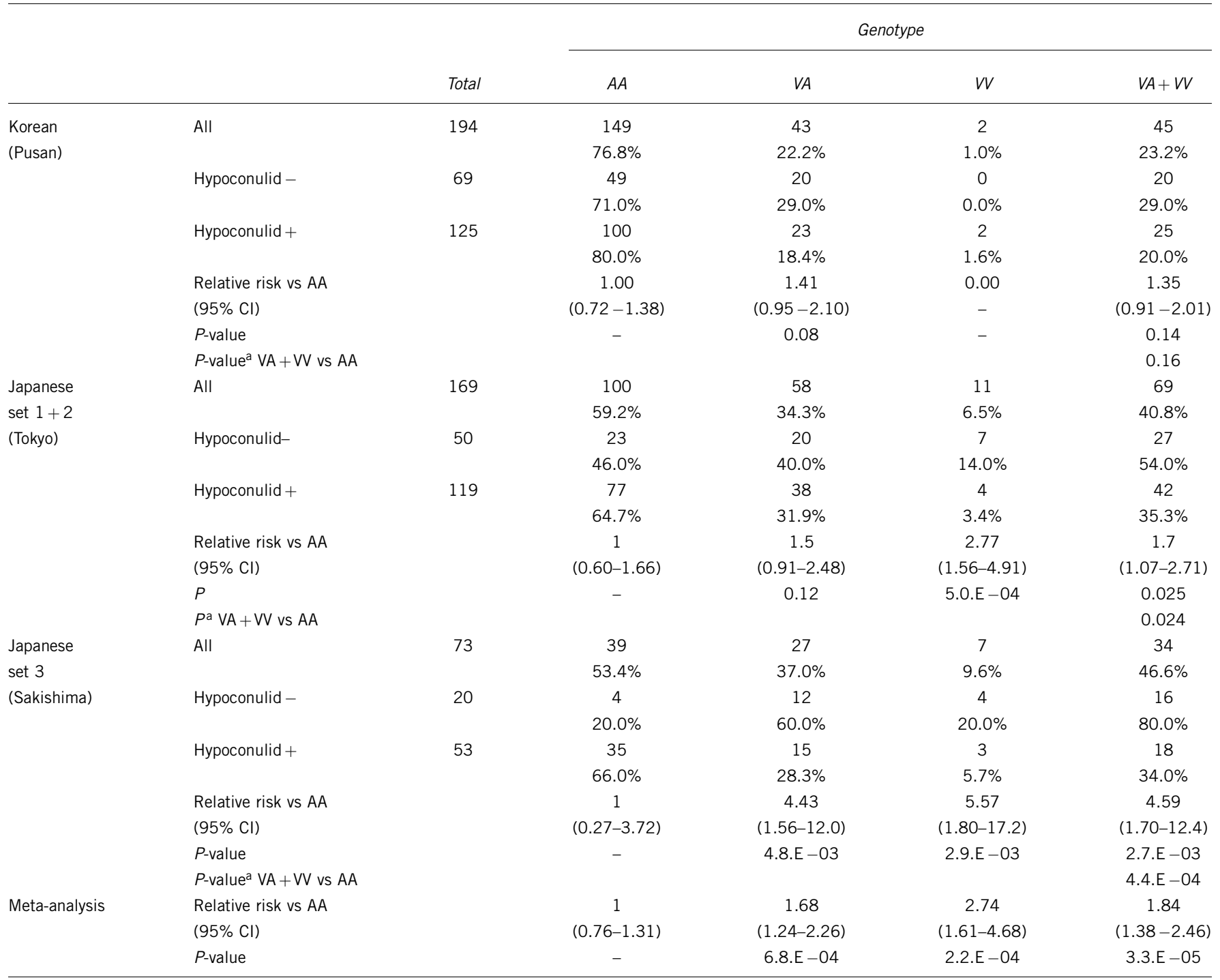

Abbreviation: $\mathrm{Cl}$, confidential interval.

aby $2 \times 2 \chi^{2}$-test.

Table 4 Correlation coefficients between the traits associated with EDAR genotype in the meta-analysis

\begin{tabular}{lcccc}
\hline & Shovel UI1 & Double shovel UI1 & Hypoconulid & Crown size all \\
\hline 370V/A (rs3827760) & 0.304 & 0.201 & 0.144 & 0.161 \\
Shovel UI1 & - & 0.264 & 0.088 & 0.313 \\
Double shovel UI1 & 0.220 & - & 0.124 & 0.192 \\
Hypoconulid & 0.049 & 0.098 & - & 0.138 \\
Crown size all & 0.284 & 0.166 & 0.119 & - \\
\hline
\end{tabular}

Upper-right, Pearson's correlation coefficients; lower-left, partial correlation coefficients between phenotypes controlling the 370V/A genotype.

Sundadonty. ${ }^{2,45,46}$ Sinodonts have significantly higher frequencies of (1) shovel-shaped UI1, (2) double-shovel-shaped UI1, (3) 1-rooted UP1, (4) UM1 enamel extensions, (5) missing/pegged/reduced UM3,
(6) LM1 defecting wrinkles, (7) 3-rooted LM1 and (8) LM2 hypoconulid. Although we could not observe all these traits as we used plaster casts, it was intriguing that the EDAR 370V/A polymorphism was associated with at least three of these eight traits (shovel-shaped incisors, double-shovel-shaped and hypoconulid). This finding suggested that EDAR variants are in part responsible for the dichotomy of Sinodonty and Sundadonty. At the same time, our analysis of partial correlation coefficients indicates the presence of unknown factors.

The global distribution of 4-cusped LM2 (the absence of hypoconulid) is complicated. It has been reported that Europeans and North Africans have a higher frequency of 4-cusped LM2 than East Asians, whereas Subsaharan Africans do not. ${ }^{3}$ These are partly consistent with a very low frequency or the absence of the EDAR 370A allele in European and North African populations. ${ }^{15,30,32}$ However, as the $370 \mathrm{~A}$ allele is also rare in Subsaharan Africans, there may be other genetic factors that affect their development of hypoconulids. 
As EDAR variants explained a small part of the variation in the presence/absence of hypoconulid in our study, it is indispensable to identify other genetic factors associated with this trait.

Studies of genome-wide SNP typing in Japanese populations have revealed that people from Okinawa fall into a different genetic cluster than people from mainland Japan, and EDAR 370V/A is one of the most differentiated nonsynonymous SNPs between the two clusters. ${ }^{47}$ This finding supports the dual-structure model for the population history of the Japanese. ${ }^{48}$ According to this model, people presently residing on mainland Japan descended from an admixture of indigenous 'Jomon' people and people from the Asian continent called 'Torai', who immigrated to Japan in the Aeneolithic 'Yayoi' period (3000-1700BP). Recent population genomics studies have proposed that the $370 \mathrm{~A}$ allele arose in Asia, and its frequency increased, especially in East Asian continental populations, due to a certain unidentified selective pressure. $^{30,31}$ In our study, the Korean subjects had a higher frequency of the $370 \mathrm{~A}$ allele than the Japanese subjects, and this finding was consistent with the dual-structure model of Japanese.

What is the true selective pressure for the EDAR 370A variant in Asian populations? This important question is still unanswered. Although our study indicated that the EDAR polymorphism affected cusp number in lower molars, shoveling and double shoveling in upper incisors and crown size, the effects of this polymorphism on these dental phenotypes are unlikely to offer any reasonable hypothesis for what the selective pressure was. The other known effect of the EDAR variant is on hair morphology, and this effect may be subject to natural or sexual selection. If this is the case, the dental phenotypes may have been resulted as by-products of the truly selected trait, hair phenotype. Taking the pleiotropic properties of EDAR into account, ${ }^{21,23,25}$ we can also speculate that the EDAR alleles affect functions in other ectodermal derivatives, such as sweat, sebaceous and salivary glands. It is possible that positive selection acted on unknown effects of the EDAR 370A variant and that the Asian phenotypes in hair and teeth are 'hitchhiking phenotypes' that occur along with another phenotype.

Here, we examined various dental traits and demonstrated that EDAR variants were associated with multiple traits. Our finding provides an example of the pleiotropic effects of one polymorphism. As technologies for the whole-genome analysis are now feasible, genetic factors, other than the EDAR variants, which affect dental morphology must be discovered in the near future. Additionally, we need to focus our attention on investigations of phenotypic variations in multiple traits in humans. Such studies will further our understanding of the molecular mechanisms that mediate morphogenesis and organogenesis, and the process of morphological evolution.

\section{CONFLICT OF INTEREST}

The authors declare no conflict of interest

\section{ACKNOWLEDGEMENTS}

We are deeply grateful to the people that participated in this study. This work was supported by the Rising Star Program for Subtropical Island Sciences at the University of the Ryukyus (to RK); KAKENHI Grant-in-Aid for Young Scientist (A) 22687023 (to RK); KAKENHI Grant-in-Aid for Scientific Research (B) 22390393 (to TY); and Heiwa Nakajima Foundation (to TY).

1 Hanihara, K. Racial characteristics in the dentition. J. Dent. Res. 46, 923-926 (1967).

2 Scott, G. R. \& Turner, C. G. I. The Anthropology of Modern Human Teeth: Dental Morphology and Its Variation in Recent Human Populations (Cambridge University Press, Cambridge, UK, 1997)
3 Hanihara, T. Morphological variation of major human populations based on nonmetric dental traits. Am. J. Phys. Anthropol 136, 169-182 (2008).

4 Hanihara, T. \& Ishida, H. Metric dental variation of major human populations. Am. J. Phys. Anthropol. 128, 287-298 (2005).

5 Mizoguchi, Y. Genetic variability in tooth crown characters: Analysis by the tetrachoric correlation method. Bull. Natl. Sci. Musium Ser. D 3, 37-62 (1977).

6 Hanihara, K., Masuda, T. \& Tanaka, T. Family studies of the shovel trait in the maxillary central incisor. J. Anthropol. Soc. Nippon 83, 107-112 (1974).

7 Garn, S. M. in. Genetics of Dental Development in the Biology of Occlusal Development (ed. McNamara, J. A. J.) 61-88 (Center for Human Growth and Development, Ann Arbor, 1977)

8 Fleischmannova, J., Matalova, E., Sharpe, P. T., Misek, I. \& Radlanski, R. J. Formation of the tooth-bone interface. J. Dent. Res. 89, 108-115 (2010).

9 Tucker, A. S. \& Sharpe, P. T. Molecular genetics of tooth morphogenesis and patterning: the right shape in the right place. J. Dent. Res. 78, 826-834 (1999).

$10 \mathrm{Bei}, \mathrm{M}$. Molecular genetics of tooth development. Curr. Opin. Genet. Dev. 19, 504-510 (2009).

11 Fraser, G. J., Hulsey, C. D., Bloomquist, R. F., Uyesugi, K., Manley, N. R. \& Streelman, J. T. An ancient gene network is co-opted for teeth on old and new jaws. PLoS Biol. 7, e31 (2009).

12 Bailleul-Forestier, I., Berdal, A., Vinckier, F., de Ravel, T., Fryns, J. P. \& Verloes, A. The genetic basis of inherited anomalies of the teeth. Part 2: syndromes with significant dental involvement. Eur. J. Med. Genet. 51, 383-408 (2008).

13 Bailleul-Forestier, I., Molla, M., Verloes, A. \& Berdal, A. The genetic basis of inherited anomalies of the teeth. Part 1: clinical and molecular aspects of non-syndromic dental disorders. Eur. J. Med. Genet. 51, 273-291 (2008).

14 Kimura, R., Yamaguchi, T., Takeda, M., Kondo, O., Toma, T., Haneji, K. et al. A common variation in EDAR is a genetic determinant of shovel-shaped incisors. Am. J. Hum. Genet. 85, 528-535 (2009).

15 Fujimoto, A., Kimura, R., Ohashi, J., Omi, K., Yuliwulandari, R., Batubara, L. et al. A scan for genetic determinants of human hair morphology: EDAR is associated with Asian hair thickness. Hum. Mol. Genet. 17, 835-843 (2008).

16 Fujimoto, A., Ohashi, J., Nishida, N., Miyagawa, T., Morishita, Y., Tsunoda, T. et al. A replication study confirmed the EDAR gene to be a major contributor to population differentiation regarding head hair thickness in Asia. Hum. Genet. 124, 179-185 (2008).

17 Yan, M., Wang, L. C., Hymowitz, S. G., Schilbach, S., Lee, J., Goddard, A. et al. Twoamino acid molecular switch in an epithelial morphogen that regulates binding to two distinct receptors. Science 290, 523-527 (2000).

18 Yan, M., Zhang, Z., Brady, J. R., Schilbach, S., Fairbrother, W. J. \& Dixit, V. M. Identification of a novel death domain-containing adaptor molecule for ectodysplasin-A receptor that is mutated in crinkled mice. Curr. Biol. 12, 409-413 (2002).

19 van der Hout, A. H., Oudesluijs, G. G., Venema, A., Verheij, J. B., Mol, B. G., Rump, P. et al. Mutation screening of the Ectodysplasin-A receptor gene EDAR in hypohidrotic ectodermal dysplasia. Eur. J. Hum. Genet. 16, 673-679 (2008).

20 Jorgenson, R. J., Dowben, J. S. \& Dowben, S. L. Autosomal dominant ectodermal dysplasia. J. Craniofac. Genet. Dev. Biol. 7, 403-412 (1987).

21 Chang, S. H., Jobling, S., Brennan, K. \& Headon, D. J. Enhanced Edar signalling has pleiotropic effects on craniofacial and cutaneous glands. PLOS ONE 4, e7591 (2009).

22 Harris, M. P., Rohner, N., Schwarz, H., Perathoner, S., Konstantinidis, P. \& Nusslein-Volhard, C. Zebrafish eda and edar mutants reveal conserved and ancestral roles of ectodysplasin signaling in vertebrates. PLoS Genet. 4, e1000206 (2008).

23 Jaskoll, T., Zhou, Y. M., Trump, G. \& Melnick, M. Ectodysplasin receptor-mediated signaling is essential for embryonic submandibular salivary gland development. Anat. Rec. A Discov. Mol. Cell. Evol. Biol. 271, 322-331 (2003).

24 Tucker, A. S., Headon, D. J., Schneider, P., Ferguson, B. M., Overbeek, P., Tschopp, J. et al. Edar/Eda interactions regulate enamel knot formation in tooth morphogenesis. Development 127, 4691-4700 (2000).

25 Monreal, A. W., Ferguson, B. M., Headon, D. J., Street, S. L. Overbeek, P. A. \& Zonana, $\mathrm{J}$. Mutations in the human homologue of mouse dl cause autosomal recessive and dominant hypohidrotic ectodermal dysplasia. Nat. Genet. 22, 366-369 (1999).

26 Kondo, S., Kuwahara, Y., Kondo, M., Naruse, K., Mitani, H., Wakamatsu, Y. et al. The medaka rs-3 locus required for scale development encodes ectodysplasin-A receptor. Curr. Biol. 11, 1202-1206 (2001).

27 Laurikkala, J., Pispa, J., Jung, H. S., Nieminen, P., Mikkola, M., Wang, X. et al. Regulation of hair follicle development by the TNF signal ectodysplasin and its receptor Edar. Development 129, 2541-2553 (2002).

28 Laurikkala, J. Mikkola, M., Mustonen, T., Aberg, T., Koppinen, P. Pispa, J et al. TNF signaling via the ligand-receptor pair ectodysplasin and edar controls the function of epithelial signaling centers and is regulated by Wnt and activin during tooth organogenesis. Dev. Biol. 229, 443-455 (2001).

29 Mustonen, T., Pispa, J., Mikkola, M. L., Pummila, M., Kangas, A. T., Pakkasjarvi, L. et al. Stimulation of ectodermal organ development by Ectodysplasin-A1. Dev. Biol. 259, 123-136 (2003).

30 Sabeti, P. C., Varilly, P., Fry, B., Lohmueller, J., Hostetter, E., Cotsapas, C. et al. Genome-wide detection and characterization of positive selection in human populations. Nature 449, 913-918 (2007).

31 Kimura, R., Fujimoto, A., Tokunaga, K. \& Ohashi, J. A practical genome scan for population-specific strong selective sweeps that have reached fixation. PLOS ONE 2, e286 (2007). 
32 The International HapMap Consortium. A haplotype map of the human genome. Nature 437, 1299-1320 (2005).

33 Carlson, C. S., Thomas, D. J., Eberle, M. A., Swanson, J. E., Livingston, R. J., Rieder, M. J. et al. Genomic regions exhibiting positive selection identified from dense genotype data. Genome Res. 15, 1553-1565 (2005).

34 Toma, T., Hanihara, T., Sunakawa, H., Haneji, K. \& Ishida, H. Metric dental diversity of Ryukyu Islanders: a comparative study among Ryukyu and other Asian populations. Anthropol. Sci. 115, 119-131 (2007).

35 Haneji, K., Hanihara, T., Sunakawa, H., Toma, T. \& Ishida, H. Nonmetric dental variation of Sakishima Islanders, Okinawa, Japan: a comparative study among Sakishima and neighboring populations. Anthropol. Sci. 115, 35-45 (2007).

36 Turner, C. G. I., Nichol, C. R. \& Scott, G. R. Scoring Procedures For Key Morphological Traits Of The Parmanent Dentition. ASU Dental Anthropology System. in Advances in Dental Anthropology (eds Kelley, M. A. \& Larsen, C. S.) 13-32 (Wiley-Liss, Inc, New York, USA, 1991).

37 Hrdlicka, A. Shovel-shaped teeth. Am. J. Phys. Anthropol. 3, 429-465 (1920).

38 Dahlberg, A. A. Material for the Establishment of Standards for Classification by Tooth Characters, Attributes, and Techniques in Morphological Studies of the Dentition (University of Chicago Press, Chicago, 1956).

39 Evans, R. \& Shaw, W. Preliminary evaluation of an illustrated scale for rating dental attractiveness. Eur. J. Orthod. 9, 314-318 (1987).

40 Tucker, A. S., Headon, D. J., Courtney, J. M., Overbeek, P. \& Sharpe, P. T. The activation level of the TNF family receptor, Edar, determines cusp number and tooth number during tooth development. Dev. Biol. 268, 185-194 (2004).
41 Pispa, J., Mustonen, T., Mikkola, M. L., Kangas, A. T., Koppinen, P., Lukinmaa, P. L. et al. Tooth patterning and enamel formation can be manipulated by misexpression of TNF receptor Edar. Dev. Dyn. 231, 432-440 (2004).

42 Bryk, J., Hardouin, E., Pugach, I., Hughes, D., Strotmann, R., Stoneking, M. et al Positive selection in East Asians for an EDAR allele that enhances NF-kappaB activation. PLoS ONE 3, e2209 (2008).

43 Mou, C., Thomason, H. A., Willan, P. M., Clowes, C., Harris, W. E., Drew, C. F. et al. Enhanced ectodysplasin-A receptor (EDAR) signaling alters multiple fiber characteristics to produce the East Asian hair form. Hum. Mutat 29, 1405-1411 (2008).

44 Nakano, M., Suzuki, A., Murakami, T. \& Takahama, Y. Tooth sizes and dentofacial forms of the orthodontic patients with tooth crowding. J. Jpn. Orthod. Soc. 52, 104-118 (1993).

45 Hanihara, K. Mongoloid dental complex in the permanent dentition. in Proceedings of the VIIIth International Congress of Anthropological and Ethnological Sciences, Vol1, 298-300 (Science Council of Japan, Tokyo, 1968).

46 Turner, 2nd C. G. Major features of Sundadonty and Sinodonty, including suggestions about East Asian microevolution, population history, and late Pleistocene relationships with Australian aboriginals. Am. J. Phys. Anthropol. 82, 295-317 (1990).

47 Yamaguchi-Kabata, Y., Nakazono, K., Takahashi, A., Saito, S., Hosono, N., Kubo, M. et al. Japanese population structure, based on SNP genotypes from 7003 individuals compared to other ethnic groups: effects on population-based association studies. Am. J. Hum. Genet. 83, 445-456 (2008).

48 Hanihara, K. Dual structure model for the population histoty of the Japanese. Japan Rev. 2, 1-33 (1991).

Supplementary Information accompanies the paper on Journal of Human Genetics website (http://www.nature.com/jhg) 